\title{
La memoria resiliente y la amnesia criptográfica: el caso de la juventud empobrecida latinoamericana
}

\section{The resilient memory and the cryptographic amnesia: the case of impoverished Latin-American youth}

\author{
Alejandro Klein \\ Universidad de Guanajuato. División de Ciencias Sociales y \\ Humanidades. León, Guanajuato, México. \\ E-mail: alejandrokleinळhotmail.com
}

\begin{abstract}
Resumen
¿Cómo es posible que se ejerza tal grado de violencia social sobre jóvenes, existiendo al mismo tiempo desempleo estructural y preocupantes tasas de deserción estudiantil? ¿Y cómo inciden estos factores en la construcción del tejido social? Este trabajo intenta responder a algunas de estas interrogantes. Se introduce el concepto de memoria criptográfica para dar a entender el proceso de resquebrajamiento del contrato social tradicional que otorgaba un lugar sustancial a los jóvenes como parte de la memoria y la transmisión generacional. Se opone al mismo el concepto de memoria resiliente por el que se reinstauran imaginarios fraternales esenciales a lo social y que la violencia neoliberal y el terrorismo de Estado intentan debilitar o anular.

Palabras clave: Adolescencia; Amnesia; Memoria; Generaciones.
\end{abstract}

\section{Correspondencia}

Blvd. Puente Milenio, 1.00I, fracción del predio San Carlos. León, Guanajuato, México. CP 37670. 


\section{Abstract}

How is it possible such degree that social violence is exerted on young people, existing at the same time structural unemployment and worrying dropout rates? Moreover, how these factors affect the construction of the social network? This paper attempts to answer some of these questions. The concept of cryptographic memory is introduced to understand the process of breaking of the traditional social contract that gave substantial place to young people as part of the memory and the generational transmission. It is opposite to the concept of resilient memory that establishes fraternal imaginary, essential to the social, and that neoliberal violence and the State terrorism try to weaken or nullify.

Keywords: Adolescence; Amnesia; Memory; Generations.
La adolescencia a golpes y los golpes sociales

Cabría preguntarse a forma de introducción: "Cómo es posible que se ejerza tal grado de violencia social sobre jóvenes, existiendo al mismo tiempo desempleo estructural y preocupantes tasas de deserción estudiantil? ¿Y cómo inciden estos factores en la construcción del tejido social?", una explicación es que las condiciones sociales que hacian posible la adolescencia se han agotado (Klein, 2006). Otra es que el lugar social del adolescente ha mutado decisivamente: de ser la "promesa" del futuro ha pasado a ser el chivo expiatorio del malestar social (Klein, 2006).

Para esto, cito la situación de la mayoría de los adolescentes de dos países latinoamericanos: Brasil y Uruguay. Una adolescente de un barrio de clase media empobrecida de Montevideo comentaba en un grupo de reflexión: "nosotros crecemos de golpe..." A lo que yo agregaba para mí mismo: "y a golpes". El golpe, a los golpes, las golpeaduras, creo que son una de las metáforas más desgraciadas, pero más atinadas, con que puedo caracterizar al vínculo del adolescente con su medio social hoy.

Es de destacar que esta metáfora del golpe refleja cómo se ha "quebrado" el contrato implícito entre la adolescencia y la red social de la modernidad keynesiana (Klein, 2006). Este contrato enunciaba de forma tácita, pero no por eso menos vigorosa, que esa sociedad era un espacio amplio y generoso, con capacidad de "albergar" a un nuevo miembro por intermedio de derechos y obligaciones, es decir, con perspectivas laborales y/o de estudio: "Trabajo y educación fueron prácticas privilegiadas para dar sentido a la identidad en la modernidad. Sin embargo, el proceso histórico pone de manifiesto la crisis de estos modos de socialización” (Cohendoz, 1999, p. 2).

A cambio se esperaba que el sujeto cumpliera ciertos requisitos imprescindibles en relación a crecimiento, madurez y experiencia, lo que lo habilitaría a ser nombrado y autonombrarse como un ser adulto-ciudadano. Es así como entiendo el concepto de moratoria de Erikson (1972): un acuerdo entre partes, en que más allá de probables enfrentamientos, existía una reconciliación inquebrantable de fondo. El sujeto creaba una historia de vida y, a cambio, la sociedad recibía la "ilusión" de poder regenerarse, transformarse y solidificarse por intermedio de sus jóvenes. 
Sin embargo las cosas se han modificado. La sociedad ya no alberga, sino que desampara. Ya no tiene (o así se intenta imponer desde el imaginario) lugar para todos y el pasaje por distintas experiencias etarias se hace difícil:

Bajo el telón del fondo de una relativa falta de cronología en el paso de las edades y de la dificultad general de llegar a la inclusión plena, la entrada al mundo adulto ocurre cada vez más tarde (lo que se extiende más la juventud), según etapas variables y desreguladas (Abramo, 2008, p. 44, la traducción es mía) ${ }^{1}$.

De esta manera, entre los grupos sociales más afectados por el neoliberalismo se encuentra la juventud empobrecida:

La mayoría de los derechos económicos, sociales, culturales y ambientales es negada a ese segmento. La educación es poco adecuada a la realidad juvenil, el acceso al trabajo -y los medios legales de generación de empleo y renta- le es vedado (Fraga; Lulianelli, 2003, p. 10).

Simultáneamente hace crisis la posibilidad de un Estado "que requiere la cooperación y el autocontrol en el ejercicio del poder privado" (Kymlicka; Norman, 1997, p. 16). Es inevitable que en estas condiciones emociones de confianza y reciprocidad sean substituidas por otras, de desconfianza y paranoia:

Nos preguntamos sobre las relaciones de odio (si no de hostilidad) existentes en la sociedad brasileña entre un grupo poblacional joven que [...] se siente "violado" su condición de lo humano y parte de la sociedad que a esta porción de la infancia y de juventud considerada "imprestable" socialmente le tiene miedo y la rechaza actuando de manera a 'eliminarla' (Takeuti, 2002, p. 28)².
De esta manera la moratoria ericksoniana ha entrado en moratoria, sugiriendo en cambio el término "pseudomoratoria" como un descriptor más efectivo de esta realidad desamparante. Esta pseudomoratoria ya no es ese dispositivo de antaño que retrasaba, tanto como preparaba la renovación del pacto social keynesiano. Hoy en día el joven si se prepara para algo, es para la inactividad, la emigración, la violencia, la exclusión o el desaliento:

Con la gradual implantación de medidas neoliberales [...] asistimos a una vehemente producción de inseguridad, miedo, pánico articulados al crecimiento del desempleo, la exclusión, la pobreza y la miseria [...] los jóvenes pobres, cuando escapan del exterminio, son los 'excluidos por excelencia', pues ni siquiera consiguen llegar al mercado de trabajo formal [...] Para los que consiguen sobrevivir, están previstos diferentes tipos de encarcelamiento. Muchos jóvenes pobres mayores de 18 años están confinados en las prisiones (Fraga; Lulianelli, 2003, p. 27-28).

A pesar de lo que indican Fraga y Lulianelli quizás es posible matizar la idea de que la exclusión es una imposibilidad de acceder al mercado laboral formal. Aunque eso sí hay hoy día una naturalización de formas marginales de inclusión laboral.

Como señalan Corea y Duschatzky (2002) este nuevo orden necesita de los integrados y de los expulsados, no en el sentido de una disfunción, sino como estructurante de lo social. Es un estar fuera del orden social, produciendo un inexistente, "un 'desaparecido' de los escenarios públicos y de intercambio. El expulsado perdió visibilidad, nombre [...] transitan por una sociedad que parece no esperar nada de ellos" (Corea; Duschatzky, 2002, p. 18).

De esta manera no es sorprendente que las instituciones que antaño incluían e integraban sedimentando un "joven conductor de acción dirigido al

1 Del original: "Sobre o pano de fundo de uma relativa descronologização do percurso das idades, e uma dificuldade geral de lograr inclusão plena, a entrada no mundo adulto se faz cada vez mais tarde (estendendo ainda mais o tempo da juventude), segundo etapas variadas e desreguladas".

2 Del original: “Interrogamo-nos sobre as relações de ódio (senão, de hostilidade) existentes na sociedade brasileira entre um segmento populacional jovem que [...] se sente "violentado" na sua condição de ser humano, e uma parte da sociedade que teme e repudia uma porção da infância e da juventude considerada "imprestável” socialmente e que atua no sentido de “eliminá-la”". 
futuro" (Fraga; Lulianelli, 2003, p. 46), se encargan hoy, por el contrario, de eliminar las condiciones y posibilidades de integración:

También la empresa fracasa en su función integradora de los jóvenes. Al elevar el nivel de las calificaciones exigidas para el ingreso, desvaloriza a una fuerza de trabajo incluso antes de que haya entrado en servicio. Jóvenes que hace veinte años se habrían integrado sin problemas en la producción, se encuentran condenados a errar de pasantía en pasantía, o de una pequeña tarea a otra (Castel, 1997, p. 408).

Aunque el joven consiga un trabajo es factible que, o quede rápidamente desempleado, o que ocupe cargos de rango netamente inferior (Fraga; Lulianelli, 2003; Tavares, 1999). Las investigaciones demuestran que se impone una educación para el desempleo (Fraga; Lulianelli, 2003; Tavares, 1999)3. De esta manera la situación de escepticismo que mantienen los jóvenes en el fondo parece ser bastante realista si consideramos las denigrantes ofertas laborales que se les ofrecen o los niveles de bajísimo salario y la inestabilidad crónica de sus contratos de trabajo. Igualmente la educación se transforma en causa de exclusión (Abramovay et al., 1999).

Esta situación consolida un sentimiento de desencanto generalizado por lo que "el discurso de los jóvenes [...] está permeado, casi todo el tiempo, por el miedo al futuro" (Fraga; Lulianelli, 2003, p. 259). El joven se educa cada vez más fuera de la escuela y no "dentro" de ella: "cuanto mayor es la edad del joven [...] menor es la frecuencia a la escuela" (Fraga; Lulianelli, 2003, p. 244).

Un correlato que no se hace esperar es la criminalización del joven que lo sindica como violento. Tanto si se identifica con el agresor como si es víctima de él, la situación permite generar un rápido, antiguo (¿e hipócrita?) convencimiento: lo que pasa a los jóvenes es culpa de los jóvenes, incapaces de dejar de ser vulgares, salvajes, descontrolados, etc. En otras palabras: si los jóvenes están tan mal es porque se lo buscan o se "lo merecen".
Se habita pues la violencia tanto reflejando "situaciones de expulsión social" (Corea; Duschatzky, 2002, p. 97) que inciden en pobreza de experiencias simbólicas (Takeuti, 2002), como por imposición de un imaginario que "violenta" lo que de por sí ya es violento.

De esta manera y progresivamente, enormes grupos de jóvenes son marginados de los sistemas de enseñanza, del trabajo y en general de los derechos de ciudadanía. La experiencia social se empobrece impidiendo que "la construcción identitaria del joven de las clases populares sea alimentada por autónomas y divergentes configuraciones del sujeto" (Fraga; Lulianelli, 2003, p. 50).

Es una sociedad que necesita que algunos de sus integrantes estén en un no-lugar "por lo que parte de sus miembros y especialmente los jóvenes de las clases sociales más carentes asumen un lugar de sobrantes" (Coutinho, 200o, p. 53, la traducción en mía). Es decir, este "no-lugar" se ha de entender como emergente de una construcción social -la adolescencia- que queda como un malestar social en tanto ya no se sabe bien cómo proceder como ella. No es negativo ni ausencia, sino que se articula a nuevas formas de funcionamiento social.

Coutinho (2000) enfatiza que los derechos de ciudadanía son sociales y que es importante la “'expectativa' de poder recibirlos" (p. 54). Esta expectativa se relaciona a mi entender al cumplimento de una "promesa" que es, como indiqué, base fundamental de la modernidad clásica y keynesiana, se haya o no cumplido:

Es preciso concebir la modernidad también desde el ángulo de la ampliación y de la universalización de la ciudadanía, o sea, concebirla como una época histórica marcada por la promesa de plena emancipación de los hombres [...] las generosas promesas de emancipación que ella creó, todavía no fueron realizadas (Coutinho, 2000, p. 68-69, la traducción es mía).

Esta falta de expectativas y de "escasez" de derechos "naturales" implica en el caso de los jóvenes la

3 Lo que contradice el argumento de los trabajos están pero lo que falta es gente calificada para ellos. Por otra parte es necesario retener la idea de que sin duda el empleo hace referencia clara a una forma de inserción social típica de la sociedad salarial. Es una referencia en los países capitalistas, pero no puede ser la única forma de concebir la inserción social. 
imposibilidad de seguir sosteniendo su "prometida" inserción social. En otras palabras, se trata quizás de la improbabilidad del acceso a los derechos y los deberes de ciudadanía, lo que se relaciona a sentimientos de inseguridad, desconcierto y desesperanza.

\section{Los procesos de des-ciudadanización y la extinción de la promesa social}

De esta manera se hacen presentes aspectos de un proceso de des-ciudadanización, que se agudiza obviamente entre los grupos sociales más pobres y desprotegidos, por lo cual el joven pierde marcos de referencia identitarios y de integración social, con extrema dificultad para que emerjan referentes sociales substitutivos. La promesa keynesiana (Klein, 2006) se debilita en tanto la adolescencia ya no pasa a ser etapa de "integración" social, sino de "extrañamiento" social. Los derechos y las obligaciones ya no pueden establecerse a priori, debido a que las mismas se formulaban y ajustaban preferentemente por intermedio del trabajo y la educación, los que están ahora cuestionados y relativizados. Es un sujeto que pierde calidad de individuo, si entendemos por "individuo" una construcción social-subjetiva inseparable del marco de ciudadanía keynesiana.

En esta situación cabe resituar el debate sobre la problemática de la "vulnerabilidad social" (Abramovay et al., 1999). A mi entender él demarca una situación en la que, al contrario de lo que indica Hanna Arendt -que define ciudadanía como "reconocimiento al derecho a tener derechos"- (Abramovay et al., 1999, p. 23), tal operatoria de reconocimiento está imposibilitada para la gran mayoría de los segmentos excluidos de la población joven latinoamericana.

Por el contrario lo que se acentúa es un proceso de identificación con el agresor (Frankel, 2002) por el cual algunos adolescentes hacen "suya" la imagen marginal que la sociedad alienta sobre él. Probablemente en el entendido de que ser lo que la sociedad desea, aunque implique una negatividad, es una manera ciertamente alienada de recibir existencia social, y que se genera al menos un punto de certeza identitaria que, aunque grave, permite cierto despliegue existencial en la cotidianeidad.

El punto fundamental es que así se acerca a una situación de indiscriminación: ser lo que el otro re- quiere que sea. Al mismo tiempo este mecanismo de defensa puede implicar ya no "tolerar" la violencia sino "ejercerla" (Freud, 1985). Esta posibilidad implica un recurso de re-individuación, por el cual los jóvenes pueden al menos responder desde un lugar de cierta "igualdad" a las situaciones a las que se los enfrenta.

No obstante habría que destacar más ampliamente cuáles son los impactos de las dificultades de integración social sobre la subjetividad adolescente y los efectos de una vulnerabilidad que pasa así a ser triple: social, familiar y psíquica. Las investigaciones sobre apego, llevadas adelante por Fonagy (2000), sugieren que una situación de maltrato puede inducir un ciclo de desarrollo severo y extremadamente perturbado. Maltrato social y maltrato familiar, en realidad, no pocas veces se realimentan dentro de un cuadro trágico:

Muchos de los jóvenes [que sufrieron violencia social] tuvieron contacto con la violencia de forma directa también en el ambiente familiar... muchos de los niños encontrados en las calles dejaron a sus familias por ser víctimas de maltratos por sus propios padres (Abramovay et al., 1999, p. 50).

De esta manera estos jóvenes presentan dificultades para ser parte de la sociedad, de su familia y dato no menor -como desarrollaré más adelante-, de su propia adolescencia. Familia, adolescencia, sociedad se le transforman en datos ajenos, externos a él mismo.

La continuidad generacional que implica la posibilidad de que al empleo del padre le suceda el empleo del hijo y que a la prosperidad del padre le suceda la del hijo se rompe indefectiblemente. Se eliminan las condiciones de seguridad básica (Giddens, 1997) y se fragilizan asímemorias generacionales arquetípicas, consolidándose lo que llamo el proceso de amnesia criptográfica. Lo amnésico del término remite a la dificultad de mantener la continuidad generacional, lo que genera en el psiquismo la presencia de criptas (Tisseron et al., 1997) que pasan a ocupar el lugar de objeto transgeneracional (Eiguer et al., 1998).

Estas criptas del psiquismo no permiten la transformación de lo anterior-generacional en forma de recuerdo y elaboración, sino que lo imponen en forma traumática y sin transformación. Por eso, desde Anzieu (1990), a mi entender se genera el 
desollamiento psíquico y social, como si jirones de "piel” psíquica y social se arrancaran sin posibilidad de transformación mediadora.

Simultáneamente se anula la obligación de investir el conjunto como continuidad y reunión de semejantes (Kaës, 1993) o se redobla tal obligación de forma imperativamente exigente (condición de hiperadaptación). Desde los aspectos referidos se impone así una no-exigencia de trabajo psíquico (Kaës, 1993), con lo cual los vínculos familiares ya no pasan por la diferenciación, sino por tareas de cuidado y protección pseudoreparatorias.

La adolescencia ${ }^{4}$ se torna un inexplorable configurando de "adolescentes sin adolescencia” (Klein, 2006). Estamos en un punto totalmente opuesto al de Aulagnier (1991), que plantea las condiciones por las cuales los jóvenes "habitan" situaciones de inclusión social desde un contrato narcisista.

Desde el neoliberalismo ocurren dos fenómenos paradojales que creo que substituyen el contrato social-narcisista keynesiano. 0 se genera un proceso de pérdida, o ambigüedad, de derechos en forma de descontractualización generalizada (es decir que se debilita el contrato de la modernidad keynesiana como un todo) o, por el contrario, aspectos referidos a obligaciones y adaptación (ya no “integración”) social se radicalizan, con lo que se impone una versión abusiva y metonimizada del contrato social-narcisista. En cualquiera de los dos casos, se acentúan aspectos instituidos del mismo que parece "expeler" a sus contratantes.

\section{La falta de proyecto social y los rituales de expiación}

De una modernidad a partir de la cual el adolescente poseía un lugar y una función social relevante se pasa a otra situación en la cual no existe proyecto social para la adolescencia. Al desaparecer el investimento previo de la sociedad, el adolescente pasa a estar situado en un presente atemporal (como el del mercado) que imprime cambios significativos en su subjetividad.

La inexistencia de estos preinvestimentos sociales junto a intentos pseudoreparadores, que se practican socialmente frente a cambios catastróficos que aparecen como inexplicables, hace que se inserte a los jóvenes en los rituales de expiación y sacrificio:

En la época moderna se están propagando una nueva forma de sacrificio: la de doble sacrificio. Se trata de un nuevo gesto de sacrificio que, al realizarse, permite crear un punto de apoyo necesario, que le faltaba, para entonces vivir, por lo menos por un momento, antes de desaparecer. Esa nueva forma de sacrificio empieza con el de víctimas precisamente elegidas [...] Seguro que resta una apelación desesperada al vínculo social (Dufour, 2005, p. 102, la traducción es mía) ${ }^{5}$.

Surgen así estructuras sociales y de pensamiento regresivas, en relación a las figuras del chivo expiatorio, chivo emisario, culpa colectiva. Es una forma -aunque fallida- de encontrarle sentido a las cosas inexplicables. Y ciertamente el mercado neoliberal, sus regulaciones y fenómenos imprimen un orden extraño e inexplicable.

El ritual del sacrificio impuesto a jóvenes que mueren simbólica o violentamente podría significar la expulsión de un "exceso" al que se responsabiliza por un estado de desequilibrio al que se anhela modificar (o se cree hacerlo) recuperando homeostasis con un procedimiento de expulsión compulsiva e irracional.

Los adolescentes retoman desde este contexto sacrificial las figuras bíblicas de chivo emisario -chivo expiatorio- (Berenstein, 1981), donde los sacrificios de expiación se realizaban con objetos (animales) destinados a desaparecer. Ritual que

4 Es importante aclarar que en esta perspectiva psicosocial que establecemos, adolescencia y juventud son conceptos epistemológicamente distintos. Mientras juventud remite a un hecho biológico, unido a la pubertad, adolescencia es una compleja construcción social entrelazada íntimamente a factores sociales, culturales y políticos. Le pido al amable lector que mantenga esta distinción.

5 Del original: “A época moderna está assim vendo se disseminar uma nova forma sacrificial: o sacrifício ao quadrado. Trata-se de um gesto sacrificial novo que, ao se perpetrar, permite criar o ponto de apoio necessário, que faltava, para enfim viver, nem que seja por um instante, antes de desaparecer. Essa nova forma sacrificial começa com o sacrifício de vítimas precisamente escolhidas [...] Com certeza resta um apelo desesperado ao laço social". 
asegura la continuidad social, un ideal cohesivo y el restablecimiento de un colectivo, que de esta manera se renueva sangrienta y circularmente.

\section{La memoria resiliente}

Sin embargo, como proceso inverso al del chivo expiatorio se verifican en diversos grupos comunitarios prácticas resilientes (Zukerfeld; Zukerfeld, 2003), que reflejan estrategias de ayuda en red y procesos de autogestión, como una forma de actualización de imaginarios y actuación de fraternidad, esenciales a la sociedad. Ubico entonces lo resiliente desde una teorización-dispositivo, que permite enfocar cómo se gestiona la presencia del otro en el conjunto social. Ya no se trata del binomio "yo o el otro"; "yo contra el otro", sino otra modalidad vincular: "yo con el otro".

Este factor de resiliencia permite consolidar formas de autogestión y protección además frente a procesos de desvalimiento. Zukerfeld y Zukerfeld (2003) señalan, desde un punto de vista psicoanalítico, que la resiliencia se relaciona a la promoción de respuestas frente al hecho traumático. Desde esta perspectiva es considerada parte de mecanismos de transformación a partir de la adversidad, con capacidad de utilizar procesos creativos inusuales.

Desde la violencia neoliberal, las experiencias del conjunto social derivadas del terrorismo de Estado, de la exclusión socioeconómica, de la injusticia y de la corrupción se han multiplicado generando condiciones de vivencia traumática (Zukerfeld; Zukerfeld, 2003). Sin embargo, al mismo tiempo las investigaciones constatan un hecho interesante: el potencial de salud de los colectivos se sobrepone y aún se fortalece sin que siempre se verifique vulnerabilidad y desarraigo extremo, ante situaciones como las descriptas.

Así es posible considerar cómo prácticas sociales y grupales decisivas habilitan un sentimiento de autoconfianza, de expansión del psiquismo, de reconocimiento del otro desde un lugar solidario (Czernikowski, 2003), contrapuesto a la cultura neoliberal desamparante o el terrorismo de Estado, cuya expresión del otro es "el enemigo" o el "peligroso".
Estas situaciones implican un entrelazamiento entre la transformación social, la solidaridad comprensiva (lo intersubjetivo) y el investimento de lo nunca investido psíquicamente (lo intrasubjetivo). Esta construcción colectiva no es sólo un decir, un relatar o un accionar. Tiene que ver también con un trabajo fundamental de la memoria. Lo resiliente apunta a una posibilidad de historización, de construcción de proyectos y porvenir entre muchos en que al mismo tiempo se sostienen fundamentos narcisistas imprescindibles (Aulagnier, 1975).

De esta manera se genera la posibilidad de "anticipar" un porvenir, es decir, construir una historia colectiva y personal simultáneamente. Desde esta perspectiva el trabajo de la memoria es la posibilidad de reinstaurar un "poder" apuntalante frente al "poder" de la violencia neoliberal y el terrorismo de Estado; de rearmar un Yo desde el Nosotros; de ofrecer indicadores de identidad; de reorganizar los conjuntos rearticulando los vínculos que sostienen al sujeto ante situaciones de ruptura catastrófica.

Desde el apuntalamiento en lo colectivo, el sujeto logra autoapuntalarse, recreando un continente psíquico capaz de mentalización y de sentir placer en la misma: "el grupo [...] mantiene el apoyo vital sobre la creencia” (Puget; Kaës, 1991, p. 149).

Esta actividad de memoria resiliente está ubicada como una actividad de reestructuración simultánea del sujeto y el conjunto, un investimento mutuo que contribuye a la restauración de la realidad externa y la realidad interna, anudando tiempo histórico y tiempo psíquico. Desde mi punto de vista es el pasaje de una situación traumatógena a un trabajo pulsional dentro del conjunto, entendiendo "pulsión" en el sentido freudiano de exigencia de actividad psíquica (Laplanche; Pontalis, 1981). Implica el pasaje de una escena cerrada y tanática a otra en la cual se libidiniza el cambio y el reapuntalamiento en los conjuntos, a su vez basándose en dos valores que cimientan resiliencia: la solidaridad y la confianza.

Pero el trabajo de la memoria y el trabajo pulsional son también trabajo de reapropiación histórica: “La construcción de un discurso popular contra-hegemónico significa la posibilidad de reapropiación o rearticulación [...] de tales elementos interpretativos, dentro del proceso de lucha” (Vasconcelos, 2000, p. 97). 
Memoria colectiva participando "del trabajo de la construcción en la memoria individual" (Puget; Kaës, 1991, p. 155), lo que permite una "comprometida reconstrucción del pasado, con énfasis en la organización de grupos sociales heterogéneos en una aprehensión y reconstrucción de identidades" (Benevides; Passos; Rauter, 2002, p. 103, la traducción es mía) ${ }^{6}$, lo que habilita y consolida un relato, una construcción de uno y muchos, que reconstruye el pasado y genera enunciados que jalonan un futuro social e individualmente viable y digno.

El desafío es enorme, pero no es imposible. La sociedad se hace en y desde los seres humanos. Y la historia y la cultura nos enseñan que hay momentos instituyentes en que el hombre puede optar por una vida mejor y un futuro prometedor. En este sentido, el neoliberalismo y las consecuencias que acarrea, de ninguna manera llegaron para quedarse.

\section{Referencias}

ABRAMO, H. W. Condição juvenil no Brasil contemporâneo. In: ABRAMO, H. W.; BRANCO, P. P. M. (Org.). Retratos da juventude brasileira: análises de uma pesquisa nacional. São Paulo: Fundação Perseu Abramo, 2008. p. 37-72.

ABRAMOVAY, M. et al. Gangues, galeras, chegados e rappers: juventude, violência e cidadania nas cidades da periferia de Brasília. Brasília, DF: Unesco; Rio de Janeiro: Garamond, 1999.

ANZIEU, D. Las envolturas psíquicas. Buenos Aires: Amorrortu, 1990.

AULAGNIER, P. La violencia de la interpretación: del pictograma al enunciado. Buenos Aires: Amorrortu, 1975 .

AULAGNIER, P. Construir(se) un pasado. Psicoanálisis: Revista de la APdeBA: Asociación Psicoanalítica de Buenos Aires, Buenos Aires, v. 13, n. 3, p. 441-468, 1991.

BENEVIDES, R.; PASSOS, E.; RAUTER, C. (Org.). Clínica e política: subjetividade e violação dos direitos humanos. Rio de Janeiro: Te Corá, 2002.
BERENSTEIN, I. Psicoanálisis de la estructura familiar: del destino a la significación. Buenos Aires: Paidós, 1981.

CASTEL, R. Las metamorfosis de la cuestión social: una crónica del salariado. Buenos Aires: Paidós, 1997.

COHENDOZ, M. Identidad joven y consumo: la globalización se ve por MTV. Revista Latina de Comunicación Social, La Laguna, n. 22, p. 1-11, out. 1999. Disponível em: <http://bit. ly/2t2HkmS>. Acesso em: 2 jun. 2009.

COREA, C.; DUSCHATZKY, S. Chicos en banda: los caminos de la subjetividad en el declive de las instituciones. Buenos Aires: Paidós, 2002.

COUTINHO, C. N. Contra a corrente: ensaios sobre democracia e socialismo. São Paulo: Cortez, 2000.

CZERNIKOWSKI, E. (Org.). Entre hermanos: sentido y efectos del vínculo fraterno. Buenos Aires: Lugar, 2003.

DUFOUR, D. R. A arte de reduzir as cabeças: sobre a nova servidão na sociedade ultraliberal. Rio de Janeiro: Companhia de Freud, 2005.

ERIKSON, E. H. Sociedad y adolescencia. Buenos Aires: Paidós, 1972.

EIGUER, A. et al. Lo generacional: abordaje en terapia familiar analítica. Buenos Aires: Amorrortu, 1998.

FONAGY, P. Apegos patológicos y acción terapéutica. Aperturas Psicoanalíticas, Madrid, n. 4, abr. 200o. Disponível em: <http://bit. ly/2sButux>. Acesso em: 23 maio 2017.

FRAGA, P. C. P.; LULIANELLI, J. A. S. (Org.). Jovens em tempo real. Rio de Janeiro: DP\&A, 2003.

FRANKEL, J. Explorando el concepto de Ferenczi de identificación con el agresor: su rol en el trauma, la vida cotidiana y la relación terapéutica. Aperturas Psicoanalíticas, Madrid, n. 11, jul. 2002. Disponível em: <http://bit.ly/2rwWGy2>. Acesso em: 23 maio 2017.

6 Del original: "reconstrução engajada do passado, com papel fundamental na organização de grupos sociais heterogéneos na sua apreensão e reconstrução de identidade”. 
FREUD, A. Psicoanálisis del desarrollo del niño y del adolescente. Buenos Aires: Paidós, 1985.

GIDDENS, A. Modernidad e identidad del yo. Madrid: Península, 1997.

KAËS, R. El grupo y el sujeto del grupo: elementos para una teoría psicoanalítica del grupo. Buenos

Aires: Amorrortu, 1993.

KLEIN, A. Adolescentes sin adolescencia: reflexiones en torno a la construcción de subjetividad adolescente bajo el contexto neoliberal. Montevideo: Psicolibros Universitario, 2006.

KYMLICKA, W.; NORMAN, W. J. El retorno del ciudadano: una revisión de la producción reciente en teoría de la ciudadanía. La Política: Revista de estudios sobre el estado y la sociedad, Barcelona, n. 3, p. 5-40, 1997.

LAPLANCHE, J.; PONTALIS, J. B. Diccionario de psicoanálisis. Madrid: Labor, 1981.
PUGET, J.; KAËS, R. Violencia de estado y psicoanálisis. Buenos Aires: Centro Editor de América Latina, 1991.

TAKEUTI, N. M. No outro lado do espelho: a fratura social e as pulsões juvenis. Brasília, DF: Relume Dumará, 2002.

TAVARES, L. Os custos sociais do ajuste neoliberal no Brasil. Santiago de Chile: Flacso, 1999.

TISSERON, S. et al. El psiquismo ante la prueba de las generaciones: clínica del fantasma. Buenos Aires: Amorrortu, 1997.

VASCONCELOS, E. Estado y políticas sociales en el capitalismo: un abordaje marxista. In: BORGIANI, E.; MONTAÑO, C. (Org.). La política social hoy. São Paulo: Cortez, 200o. p. 126-135.

ZUKERFELD, R.; ZUKERFELD, Z. Z. Procesos terciarios. Aperturas de Psicoanálisis, Madrid, n. 14, jul. 2003. Disponível em: <http://bit. ly/2rwQZ3p>. Acesso em: 23 maio 2013.
Recibido: 08/01/2016

Re-presentado: 04/05/2017

Aceptado: 15/05/2017 